\title{
Characteristics of older adults using patient web portals to view their DXA results
}

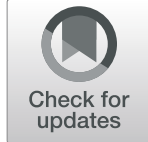

\author{
Stephanie Edmonds ${ }^{1,2^{*}}$ (D), Yiyue Lou ${ }^{3}$, Brandi Robinson ${ }^{4}$, Peter Cram ${ }^{5,6}$, Douglas W. Roblin ${ }^{7}$, Nicole C. Wright ${ }^{8}$, \\ Kenneth Saag ${ }^{9}$ and Fredric D. Wolinsky ${ }^{1,10,11}$
}

\begin{abstract}
Background: Sharing test results with patients via patient web portals is a new trend in healthcare. No research has been done examining patient web portal use with bone density test results. The objective of our study was to identify patient characteristics associated with the use of patient web portals to view their bone density test results.

Methods: A secondary analysis of data from a pragmatic randomized controlled trial of 7749 participants $\geq 50$ years old that had presented for a dual energy X-ray absorptiometry (DXA) bone density test. Patients were interviewed at enrollment and 12 weeks later. Multivariable logistic regression identified patient characteristics that differentiated those who used the web portal from those who did not.

Results: Our sample included 4669 patients at the two (University of lowa [UI], and Kaiser Permanente of Georgia [KPGA]) clinical sites that had patient web portals. Of these patients, 3399 (72.8\%) reported knowing their test results 12 weeks post-DXA, with 649 (13.9\%) reporting that they viewed their DXA results using the web portal. Web portal users were more likely to be from UI than KPGA, and were younger, more educated, had higher health literacy, had osteopenia, and had the same sex as their referring physician (all $p<0.05$ ).

Conclusion: Only $19.1 \%$ of the 3399 patients who knew their DXA results used the available patient web portals to find out about them. Web portal users differed from non-users on several characteristics. This suggests that simply making patient web portals available for use may not be sufficient to appreciably enhance patient awareness of their test results. Based on these findings, a better understanding of the reasons why older, less educated, and less activated patients do not access their test results through patient web portals is needed.
\end{abstract}

\section{Introduction}

In 2009, the Centers for Medicare and Medicaid Services (CMS) established the Electronic Health Records Incentive Programs "to promote the adoption and meaningful use of interoperable health information technology (HIT) and qualified electronic health records (EHRs)" [1]. One HIT approach for achieving this goal is sharing information in the EHR with patients via web portals. The advantages of providing patients with access to their test results are numerous, including fostering self-efficacy and patient activation as well as preventing test results from falling-through-the cracks, which is a common medical error and a threat to patient safety [2].

\footnotetext{
* Correspondence: stephanie-edmonds@uiowa.edu

${ }^{1}$ Carver College of Medicine, Department of Internal Medicine, University of lowa, 200 Newton Rd. 5231 WL, lowa City, IA 52242, USA

${ }^{2}$ CADRE, lowa City VA Health System, lowa City, IA, USA

Full list of author information is available at the end of the article
}

For this reason, the Joint Commission established goals for communicating test results [3]. Patient portals, like MyChart from Epic, is one method patients can access their test results. Patient web portals have been shown to improve patient satisfaction and health care quality [4-10].

A commonly performed test among older adults is dual energy $x$-ray absorptiometry (DXA), which is the standard screening tool for identifying patients at increased risk for hip or other fragility fractures. But even when a diagnosis of osteoporosis is made from the DXA, patients do not always receive appropriate pharmacotherapy consistent with treatment guidelines [11]. Indeed, one study found that two-thirds of patients with DXA results indicating osteoporosis did not receive appropriate pharmacotherapy. And using chart review methods, another study revealed among $23 \%$ of abnormal DXA results there was no evidence that the patient's clinician had reviewed their DXA results or communicated

(c) The Author(s). 2019 Open Access This article is distributed under the terms of the Creative Commons Attribution 4.0 International License (http://creativecommons.org/licenses/by/4.0/), which permits unrestricted use, distribution, and reproduction in any medium, provided you give appropriate credit to the original author(s) and the source, provide a link to the Creative Commons license, and indicate if changes were made. The Creative Commons Public Domain Dedication waiver (http://creativecommons.org/publicdomain/zero/1.0/) applies to the data made available in this article, unless otherwise stated. 
them to their patients [11]. One way to increase patient access to their DXA results would be to use web portals to communicate test results directly to patients. To our knowledge, however, there have not been any studies of patients' use of web portals for learning about their DXA results. Sharing DXA results with patients via web portals may also activate them to follow-up with their health care provider, especially if their results are abnormal.

Web portals are relatively new in medicine. A recent systematic review found low use rates for patient web portals across a number of studies [12]. The main reasons given included concerns about confidentiality, poor awareness of web portals, and inadequate user friendliness. Overall, patients who did use web portals as part of the health care were typically younger, and more likely to be White, affluent, women with chronic disease [12].

Our objective was to examine patient and clinical factors associated with the use of web portals to review their DXA results. We did this using data from the Patient Activation after DXA Result Notification clinical trial (PAADRN- https://clinicaltrials.gov/ct2/show/ NCT01507662), Identifying the characteristics of web portal users, specifically for DXA results, may provide the information needed to inform practitioners about the appropriate ways to communicate DXA results, as well as provide insight into how best to craft interventions to increase web portal use.

\section{Methods}

\section{PAADRN study}

This manuscript describes a secondary data analysis of data from the PAADRN study, a pragmatic, randomized controlled trial conducted at the DXA clinics of three medical centers in the United States (U.S.) - the University of Iowa (UI), the University of Alabama at Birmingham (UAB), and Kaiser Permanente of Georgia (KPGA) [13]. PAADRN's main purpose was to examine the effects a tailored DXA result notification letter accompanied by a bone-health educational brochure mailed to patients on guideline-concordant osteoporosis pharmacotherapy., Eligible patients were those presenting for DXA who were 50 years old or older and could read and speak English. We excluded patients who had significant cognitive impairments which would prevent them from providing informed consent, prisoners, and those with visual or hearing impairments. Institutional Review Boards from all three sites approved the study and we collected written or verbal informed consent per institutional policy.

\section{Survey instrument}

As previously described [13], study-specific RAs asked participants questions about their demographics, bone and general health histories, health literacy, subjective numeracy $(\alpha=0.811)$ [14], preference for self-care $(\alpha=0.771)$ [15], patient activation (PAM, $\alpha=0.661)$ [16], and measures of their osteoporosis-related knowledge $(\alpha=0.685)[17,18]$, health beliefs $(\alpha=0.737)$ [19], and self-efficacy $(\alpha=0.958)$ [20]. About 12-weeks later, trained interviewers from the Iowa Social Science Research Center re-contacted patients by telephone and asked them a series of questions related to their DXA. In particular, patients were asked if they knew their DXA results, and if they did, how it was that they came to know about their DXA results. Patients could select more than one response option. The response options for this latter question were:

- meeting with their provider in person

- talking to their provider over the phone

- exchanging emails with their provider

- receiving a mailed letter with their results (a letter was mailed to all intervention participants)

- seeing their results on an electronic medical record or portal

- getting their results from the DXA technician

- or by some other means.

Once participants identified the methods by which they received their DXA results, the interviewers then asked them to indicate the order in which they received the results (e.g. which method was the first, second, third, etc., by which you learned of your results). A more detailed description of the interview protocol is available elsewhere [13].

\section{Analytic sample}

We limited the current analysis to PAADRN participants from UI and KPGA because both sites had web portals available at the time of PAADRN enrollment. UAB did not have a web portal for the entire study period, and thus UAB patients were excluded. Web portal assignment was not randomized in this study. Both UI (since June 2010) and KPGA (since 2005) offered patients access to Epic's (Verona, WI) web portal (known as MyHealthManager for KPGA and MyChart for UI). Of the 3185 UI patients who were potentially eligible for PAADRN, administrative records indicated that $62.6 \%$ had activated their MyChart accounts. Similarly, of 15,642 potentially eligible patients at KPGA, administrative records indicated that $65 \%$ had activated their MyHealthManager accounts. We further limited the analytic sample to PAADRN participants who reported knowing what their test results were at the time of their 12-week survey.

\section{Statistical analysis}

We first used bivariable analysis to compare all web portal users with all non-web portal users. This included 
a comparison of demographic characteristics (clinical site, age, sex, race: white vs. not white, and education), patient health beliefs (health literacy, numeracy, patient activation, preference for self-care [14]), health characteristics (self-reported history of low bone density or osteoporosis, self-rated health, and a number of additional chronic conditions), DXA ordering provider characteristics (intervention assignment, provider's gender, and whether the provider was the same sex as the patient). Continuous variables (age, health literacy, numeracy, patient activation scores, and self-care preferences) were categorized and some categorical variables (race, education, number of chronic conditions) were collapsed to improve interpretation.

Next, among web portal users we distinguished between three groups. The first group of web portal users included those who reported only learning about their results from the web portal. The second group included those who used the web portal as their first method to learn of their results, but also learned about their results later on from another source. The third group included those who first learned about their results from another method, but also learned about their results subsequently from the web portal. We compared these three groups on the demographic characteristics, patient health beliefs, health, and provider characteristics to determine whether pooling these groups was appropriate.

Finally, we used multivariable logistic regression with any web portal use as the outcome, and all of the predictors that were used in the bivariable analyses. Adjusted odds ratios (AORs) and 95\% CIs were calculated for each predictor. Case-wise deletion was used to address missing data. To examine the heterogeneity of treatment effects (HTEs) involving study site, we conducted sensitivity analyses by including interactions of site vs. all of the other predictors one at a time in the adjusted models. All analyses were performed using SAS 9.4, Cary, NC.

\section{Results}

\section{Study population}

PAADRN's CONSORT flow chart and descriptive baseline data for all 7749 participants can be found elsewhere [21]. Of the 4669 patients enrolled at UI and KPGA, 4005 (85.8\%) completed the 12-week interview, and $84.9 \%$ of those $(N=3399)$ knew the results of their DXA (Table 1). Overall, $13.9 \%(n=649)$ of participants used the web portal to view their DXA results.

\section{Unadjusted associations with web portal use}

Participants who knew their DXA results and reported using the web portal as one method of viewing their results were more likely to be from UI, younger, White, more highly educated, have higher health literacy and numeracy, have higher patient activation scores, have higher health information-seeking preferences. Additionally, web portal users differed on health-related characteristics such as those with history of osteopenia or osteoporosis, better self-rated health, and a female provider or a provider of the same gender than participants who did not use the web portal to view their DXA results (Table 1).

\section{Method of learning of DXA result}

Of the 649 participants who used the web portal, 192 (29.6\%) learned of their DXA results only through the web portal, 230 (35.4\%) learned of their DXA results by another method as well but through the web portal first, and 227 (35.0\%) learned of their DXA results from another method first but viewed the result later via the web portal (Table 2). The three groups did not differ statistically on any characteristic; thus, it was appropriate for us to combine all three groups into one "web portal use' group.

\section{Adjusted associations with web portal use}

The multivariable logistic regression analyses (Table 3) revealed that overall, web portal use was more likely for patients who were at UI $(\mathrm{REF}=\mathrm{KP}, \mathrm{AOR}=1$ / $0.39=2.56, p$-value $<0.001)$, younger than $75(\mathrm{REF}=$ $65-75$, AOR $=1 / 0.67=1.49, p$-value $=0.011)$, more educated (REF=High school graduate or less, AOR = 1.56 to 1.81 , all $p$-values $<0.002)$, had higher health literacy $\quad(\mathrm{AOR}=1.50, \quad p$-value $=0.014), \quad$ self-reported osteopenia $\quad(\mathrm{REF}=\mathrm{Normal}, \quad \mathrm{AOR}=1.45, \quad p$-value $=$ $0.001)$, and were of the same gender at their provider $(\mathrm{AOR}=1.41, p$ value $=0.006)$. Within sites, the associations were again comparable, although $p$ values were less significant due to the reduced sample sizes. The C-statistic (AUC) for our logistic regression model is 0.704, which indicates that it is a good model. Also, the p-value for Hosmer-Lemeshow test is 0.270, suggesting that there is no lack of fit.

\section{Discussion}

We used data from a large multi-site, pragmatic randomized controlled trial to examine which patients learned of their DXA results using available patient web portals. Our analysis revealed that most participants $(80.9 \%)$ never used the patient web portals to view their DXA results. We also found that portal use was more common among patients who were at UI, younger than 75 , more educated, had higher health literacy, self-reported osteopenia, and had a provider of the same gender.

These findings are comparable to reports from other studies that have found web portal use rates ranging from 16 to $35 \%$ [22-28]. Direct comparisons are 
Table 1 Characteristics of those viewing DXA results via web portal vs. those not viewing DXA results via web portal among patients reported knowing their DXA results $(N=3399)$

\begin{tabular}{|c|c|c|c|}
\hline Characteristic & $\begin{array}{l}\text { Viewed DXA results via web portal, } \\
N(\%)=649(19.1 \%)\end{array}$ & $\begin{array}{l}\text { Did not view DXA results via web portal } \\
N(\%)=2750(80.9 \%)\end{array}$ & $P$-value \\
\hline \multicolumn{4}{|l|}{ Demographics } \\
\hline \multicolumn{4}{|l|}{ Site } \\
\hline UI & $385(29.26 \%)$ & $931(70.74 \%)$ & \multirow[t]{2}{*}{$<0.001$} \\
\hline KPGA & $264(12.67 \%)$ & $1819(87.33 \%)$ & \\
\hline \multicolumn{4}{|l|}{ Age } \\
\hline Mean (SD) & $64.4(7.4)$ & $66.5(8.1)$ & $<0.001$ \\
\hline$<65$ & $313(22.66 \%)$ & $1068(77.34 \%)$ & \multirow[t]{3}{*}{$<0.001$} \\
\hline $65-74$ & $270(18.38 \%)$ & $1199(81.62 \%)$ & \\
\hline$>75$ & $66(12.02 \%)$ & $483(87.98 \%)$ & \\
\hline \multicolumn{4}{|l|}{ Gender of patient } \\
\hline Male & $113(17.15 \%)$ & $546(82.85 \%)$ & \multirow[t]{2}{*}{0.157} \\
\hline Female & $536(19.56 \%)$ & $2204(80.44 \%)$ & \\
\hline \multicolumn{4}{|l|}{ Race } \\
\hline White & $570(21.19 \%)$ & $2120(78.81 \%)$ & \multirow[t]{2}{*}{$<0.001$} \\
\hline Non-white & $79(11.14 \%)$ & $630(88.86 \%)$ & \\
\hline \multicolumn{4}{|l|}{ Education } \\
\hline High school or less & $88(10.92 \%)$ & $718(89.08 \%)$ & \multirow[t]{4}{*}{$<0.001$} \\
\hline Some college & 197 (18.02\%) & 896 (81.98\%) & \\
\hline Completed college & $157(21.3 \%)$ & $580(78.7 \%)$ & \\
\hline Graduate school & $204(27.79 \%)$ & $530(72.21 \%)$ & \\
\hline \multicolumn{4}{|c|}{ Patient health personal characteristics } \\
\hline \multicolumn{4}{|l|}{ Health Literacy } \\
\hline Low & $54(12.08 \%)$ & $393(87.92 \%)$ & \multirow[t]{2}{*}{$<0.001$} \\
\hline High & $592(20.27 \%)$ & $2328(79.73 \%)$ & \\
\hline \multicolumn{4}{|l|}{ Subjective Numeracy } \\
\hline Low & $160(13.86 \%)$ & 994 (86.14\%) & \multirow[t]{3}{*}{$<0.001$} \\
\hline Medium & $213(18.85 \%)$ & 917 (81.15\%) & \\
\hline High & $273(25.11 \%)$ & $814(74.89 \%)$ & \\
\hline \multicolumn{4}{|l|}{ Patient Activation Score } \\
\hline Level 1 (0-42.5) & $50(15.2 \%)$ & $279(84.8 \%)$ & \multirow[t]{4}{*}{0.003} \\
\hline Level 2 (47.4-52.9) & $100(16.05 \%)$ & $523(83.95 \%)$ & \\
\hline Level 3 (56.4-66.0) & $302(19.28 \%)$ & $1264(80.72 \%)$ & \\
\hline Level 4 (68.5-100) & $194(22.61 \%)$ & $664(77.39 \%)$ & \\
\hline \multicolumn{4}{|l|}{ Krantz Information Scale } \\
\hline Low & $164(14.84 \%)$ & $941(85.16 \%)$ & \multirow[t]{3}{*}{$<0.001$} \\
\hline Medium & $236(20.36 \%)$ & $923(79.64 \%)$ & \\
\hline High & $246(22.18 \%)$ & $863(77.82 \%)$ & \\
\hline \multicolumn{4}{|l|}{ Krantz Behavioral Scale } \\
\hline Low & $187(15.7 \%)$ & 1004 (84.3\%) & \multirow[t]{3}{*}{$<0.001$} \\
\hline Medium & $193(18.16 \%)$ & $870(81.84 \%)$ & \\
\hline High & $261(23.66 \%)$ & $842(76.34 \%)$ & \\
\hline
\end{tabular}


Table 1 Characteristics of those viewing DXA results via web portal vs. those not viewing DXA results via web portal among patients reported knowing their DXA results $(N=3399)$ (Continued)

\begin{tabular}{|c|c|c|c|}
\hline Characteristic & $\begin{array}{l}\text { Viewed DXA results via web portal, } \\
N(\%)=649(19.1 \%)\end{array}$ & $\begin{array}{l}\text { Did not view DXA results via web portal } \\
N(\%)=2750(80.9 \%)\end{array}$ & $P$-value \\
\hline \multicolumn{4}{|l|}{ Participant health characteristics } \\
\hline \multicolumn{4}{|l|}{ History of Osteoporosis } \\
\hline Normal & $320(16.04 \%)$ & 1675 (83.96\%) & \multirow[t]{3}{*}{$<0.001$} \\
\hline Osteopenia & $206(26.68 \%)$ & $566(73.32 \%)$ & \\
\hline Osteoporosis & $123(19.46 \%)$ & $509(80.54 \%)$ & \\
\hline \multicolumn{4}{|l|}{ Self-rated Health } \\
\hline Poor & $17(17.17 \%)$ & $82(82.83 \%)$ & \multirow[t]{5}{*}{0.007} \\
\hline Fair & $68(15.96 \%)$ & $358(84.04 \%)$ & \\
\hline Good & $215(17.68 \%)$ & $1001(82.32 \%)$ & \\
\hline Very good & 245 (19.84\%) & $990(80.16 \%)$ & \\
\hline Excellent & $104(24.94 \%)$ & $313(75.06 \%)$ & \\
\hline \multicolumn{4}{|l|}{ Number of Chronic Conditions } \\
\hline None & $305(19.28 \%)$ & 1277 (80.72\%) & \multirow[t]{3}{*}{0.485} \\
\hline $1-2$ & $313(19.31 \%)$ & $1308(80.69 \%)$ & \\
\hline$>2$ & $31(15.82 \%)$ & $165(84.18 \%)$ & \\
\hline \multicolumn{4}{|l|}{ Provider characteristics } \\
\hline \multicolumn{4}{|l|}{ Intervention } \\
\hline Intervention & $316(17.86 \%)$ & $1453(82.14 \%)$ & \multirow[t]{2}{*}{0.057} \\
\hline Usual care & $333(20.43 \%)$ & $1297(79.57 \%)$ & \\
\hline \multicolumn{4}{|l|}{ Patient Provider's Gender } \\
\hline Male & $208(16.72 \%)$ & $1036(83.28 \%)$ & \multirow[t]{2}{*}{0.007} \\
\hline Female & $441(20.46 \%)$ & 1714 (79.54\%) & \\
\hline \multicolumn{4}{|l|}{ Gender of patient and provider } \\
\hline Female providers and female patients & $398(21.37 \%)$ & $1464(78.63 \%)$ & \multirow[t]{4}{*}{0.001} \\
\hline Female providers and male patients & $43(14.68 \%)$ & $250(85.32 \%)$ & \\
\hline Male providers and male patients & $70(19.13 \%)$ & $296(80.87 \%)$ & \\
\hline Male providers and female patients & $138(15.72 \%)$ & 740 (84.28\%) & \\
\hline
\end{tabular}

difficult, however, because of the varying definitions of web portal use including whether patients were only registered [22, 26, 28], or if they logged in, or if they activated their account [23-25, 27]. Our study was restricted to patients who reported knowing their DXA results and said that they viewed their DXA results using the web portal. We were not, however, able to know if those who did not use the web portal to view their DXA results used the portal for other purposes.

Low rates of web portal use may be due to patients preferring other forms of communication with their providers for getting their test results. While web portals are strongly endorsed [1], patients may not like them [29]. A study of patient preferences for DXA result notification found that $18 \%$ of the patients considered it unacceptable to provide either normal or abnormal DXA results over a secure web portal [29]. Perhaps when patients become more familiar with web portals, their acceptance of web portal notification of test results will increase. Learning more about patient's perceptions of web portals may also lead to increased use, especially among older adults.

We found that after adjusting for other factors, patients $>75$ years old had $67 \%$ lower odds of using the web portal to view their DXA results. This is consistent with most prior studies of web portal use [22], although Krist et al. reported an anomalous finding that patients 60-69 years old were more likely to use the web portal than patients $18-59$ or 70 years old or older [24]. It has been suggested that older patients actually may be more engaged to use a web portal if they have more chronic diseases, office visits, and diagnostic tests to be informed about [24, 30, 31]. For example, Ancker et al. did find that those with chronic conditions were more likely to 
Table 2 Characteristics of web portal users who first learned their DXA results through different sources $(N=649)$

\begin{tabular}{|c|c|c|c|c|}
\hline Characteristic & $\begin{array}{l}\text { Web portal only, } \\
N(\%)=192(29.6 \%)\end{array}$ & $\begin{array}{l}\text { Web portal first, } \\
N(\%)=230(35.4 \%)\end{array}$ & $\begin{array}{l}\text { Web portal, but not first } \\
\text { method reported, } \\
\mathrm{N}(\%)=227(35.0 \%)\end{array}$ & $P$-value \\
\hline \multicolumn{5}{|l|}{ Demographics } \\
\hline \multicolumn{5}{|l|}{ Site } \\
\hline $\mathrm{UI}$ & 117 (30.39\%) & 132 (34.29\%) & 136 (35.32\%) & \multirow[t]{2}{*}{0.742} \\
\hline KPGA & $75(28.41 \%)$ & $98(37.12 \%)$ & 91 (34.47\%) & \\
\hline \multicolumn{5}{|l|}{ Age } \\
\hline Mean (SD) & $64.9(8.2)$ & $64.1(7.1)$ & $64.3(7.2)$ & 0.503 \\
\hline$<65$ & 91 (29.07\%) & $113(36.1 \%)$ & 109 (34.82\%) & \multirow[t]{3}{*}{0.290} \\
\hline $65-74$ & $74(27.41 \%)$ & 99 (36.67\%) & 97 (35.93\%) & \\
\hline$>75$ & 27 (40.91\%) & $18(27.27 \%)$ & $21(31.82 \%)$ & \\
\hline \multicolumn{5}{|l|}{ Gender of Patient } \\
\hline Male & 35 (30.97\%) & 37 (32.74\%) & 41 (36.28\%) & \multirow[t]{2}{*}{0.804} \\
\hline Female & 157 (29.29\%) & 193 (36.01\%) & $186(34.7 \%)$ & \\
\hline \multicolumn{5}{|l|}{ Race } \\
\hline White & 171 (30\%) & 202 (35.44\%) & 197 (34.56\%) & \multirow[t]{2}{*}{0.777} \\
\hline Non-white & $21(26.58 \%)$ & $28(35.44 \%)$ & 30 (37.97\%) & \\
\hline \multicolumn{5}{|l|}{ Education } \\
\hline High school or less & $28(31.82 \%)$ & $30(34.09 \%)$ & 30 (34.09\%) & \multirow[t]{4}{*}{0.907} \\
\hline Some college & $53(26.9 \%)$ & 70 (35.53\%) & $74(37.56 \%)$ & \\
\hline Completed college & 47 (29.94\%) & 60 (38.22\%) & $50(31.85 \%)$ & \\
\hline Graduate school & $63(30.88 \%)$ & 69 (33.82\%) & 72 (35.29\%) & \\
\hline \multicolumn{5}{|c|}{ Patient health personal characteristics } \\
\hline \multicolumn{5}{|l|}{ Health Literacy } \\
\hline Low & $13(24.07 \%)$ & 19 (35.19\%) & $22(40.74 \%)$ & \multirow[t]{2}{*}{0.560} \\
\hline High & $178(30.07 \%)$ & $210(35.47 \%)$ & $204(34.46 \%)$ & \\
\hline \multicolumn{5}{|l|}{ Subjective Numeracy } \\
\hline Low & $51(31.88 \%)$ & $60(37.5 \%)$ & 49 (30.63\%) & \multirow[t]{3}{*}{0.707} \\
\hline Medium & $61(28.64 \%)$ & $77(36.15 \%)$ & 75 (35.21\%) & \\
\hline High & $79(28.94 \%)$ & $92(33.7 \%)$ & $102(37.36 \%)$ & \\
\hline \multicolumn{5}{|l|}{ Patient Activation Score } \\
\hline Level 1 (0-42.5) & $24(48 \%)$ & $16(32 \%)$ & $10(20 \%)$ & \multirow[t]{4}{*}{0.031} \\
\hline Level 2 (47.4-52.9) & $24(24 \%)$ & $36(36 \%)$ & $40(40 \%)$ & \\
\hline Level 3 (56.4-66.0) & $92(30.46 \%)$ & 99 (32.78\%) & $111(36.75 \%)$ & \\
\hline Level 4 (68.5-100) & $51(26.29 \%)$ & $78(40.21 \%)$ & 65 (33.51\%) & \\
\hline \multicolumn{5}{|l|}{ Krantz Information Scale } \\
\hline Low & $48(29.27 \%)$ & $50(30.49 \%)$ & $66(40.24 \%)$ & \multirow[t]{3}{*}{0.385} \\
\hline Medium & $67(28.39 \%)$ & 87 (36.86\%) & $82(34.75 \%)$ & \\
\hline High & 77 (31.3\%) & $92(37.4 \%)$ & $77(31.3 \%)$ & \\
\hline \multicolumn{5}{|l|}{ Krantz Behavioral Scale } \\
\hline Low & $51(27.27 \%)$ & $63(33.69 \%)$ & 73 (39.04\%) & \multirow[t]{3}{*}{0.652} \\
\hline Medium & $61(31.61 \%)$ & 69 (35.75\%) & 63 (32.64\%) & \\
\hline High & 78 (29.89\%) & 97 (37.16\%) & 86 (32.95\%) & \\
\hline
\end{tabular}




\begin{tabular}{|c|c|c|c|c|}
\hline Characteristic & $\begin{array}{l}\text { Web portal only, } \\
N(\%)=192(29.6 \%)\end{array}$ & $\begin{array}{l}\text { Web portal first, } \\
N(\%)=230(35.4 \%)\end{array}$ & $\begin{array}{l}\text { Web portal, but not first } \\
\text { method reported, } \\
\mathrm{N}(\%)=227(35.0 \%)\end{array}$ & $P$-value \\
\hline \multicolumn{5}{|l|}{ Participant health characteristics } \\
\hline \multicolumn{5}{|l|}{ History of Osteoporosis } \\
\hline Normal & $94(29.38 \%)$ & 107 (33.44\%) & 119 (37.19\%) & \multirow[t]{3}{*}{0.713} \\
\hline Osteopenia & $63(30.58 \%)$ & $74(35.92 \%)$ & $69(33.5 \%)$ & \\
\hline Osteoporosis & $35(28.46 \%)$ & 49 (39.84\%) & 39 (31.71\%) & \\
\hline \multicolumn{5}{|l|}{ Self-rated Health } \\
\hline Poor & $5(29.41 \%)$ & $5(29.41 \%)$ & $7(41.18 \%)$ & \multirow[t]{5}{*}{0.488} \\
\hline Fair & $26(38.24 \%)$ & $20(29.41 \%)$ & $22(32.35 \%)$ & \\
\hline Good & $64(29.77 \%)$ & $84(39.07 \%)$ & $67(31.16 \%)$ & \\
\hline Very good & $73(29.8 \%)$ & $84(34.29 \%)$ & $88(35.92 \%)$ & \\
\hline Excellent & $24(23.08 \%)$ & $37(35.58 \%)$ & $43(41.35 \%)$ & \\
\hline \multicolumn{5}{|l|}{ Number of Chronic Conditions } \\
\hline None & $86(28.2 \%)$ & $112(36.72 \%)$ & 107 (35.08\%) & \multirow[t]{3}{*}{0.573} \\
\hline $1-2$ & $93(29.71 \%)$ & $108(34.5 \%)$ & $112(35.78 \%)$ & \\
\hline$>2$ & $13(41.94 \%)$ & $10(32.26 \%)$ & $8(25.81 \%)$ & \\
\hline \multicolumn{5}{|l|}{ Provider characteristics } \\
\hline \multicolumn{5}{|l|}{ Intervention Assignment } \\
\hline Intervention & $88(27.85 \%)$ & $121(38.29 \%)$ & 107 (33.86\%) & \multirow[t]{2}{*}{0.323} \\
\hline Usual care & $104(31.23 \%)$ & 109 (32.73\%) & $120(36.04 \%)$ & \\
\hline \multicolumn{5}{|l|}{ Patient Provider's Gender } \\
\hline Male & $68(32.69 \%)$ & $68(32.69 \%)$ & $72(34.62 \%)$ & \multirow[t]{2}{*}{0.435} \\
\hline Female & $124(28.12 \%)$ & $162(36.73 \%)$ & 155 (35.15\%) & \\
\hline \multicolumn{5}{|l|}{ Gender of patient and provider } \\
\hline Female providers and female patients & $114(28.64 \%)$ & $145(36.43 \%)$ & 139 (34.92\%) & \multirow[t]{4}{*}{0.799} \\
\hline Female providers and male patients & $10(23.26 \%)$ & $17(39.53 \%)$ & $16(37.21 \%)$ & \\
\hline Male providers and male patients & $25(35.71 \%)$ & $20(28.57 \%)$ & $25(35.71 \%)$ & \\
\hline Male providers and female patients & $43(31.16 \%)$ & $48(34.78 \%)$ & $47(34.06 \%)$ & \\
\hline
\end{tabular}

register for a patient web portal [22]. While we did not find a significant difference in web portal use based on the number of patient comorbidities, we did find that those who self-reported osteopenia had $45 \%$ higher odds of viewing their DXA via their web portal results than those with normal bone density. Patients with a history of osteopenia may be more anxious and thus more motivated to get their DXA results.

Unlike other studies [22-24, 26-28], we did not find that race or sex differed between web portal users and non-users. This may be due to the low number of nonWhites, especially at UI, and to having men included in our sample given that osteoporosis is general associated with White females. One of our most interesting findings was that patients with the same gender as their provider had $41 \%$ greater odds of using the web portal than those with a provider of a different gender. To our knowledge, only one other study has examined clinician characteristics related to patient web portal use, and they also found that patients with female clinicians were 37\% more likely to use a web portal [24]. Others have shown that women are more likely to be earlier adopters of web portals than men [22-24], and clinician adoption of web portals may stimulate patient use [24].

Consistent with other studies [12, 26, 32], we found that more educated patients had between 56 and $81 \%$ greater odds and those with higher health literacy had $50 \%$ greater odds of using the web portal to view their DXA results than those with lower education and health literacy. We expected this finding because activating, accessing, and navigating a web portal requires patients to read health-related terms. If web portals are to be emphasized and encouraged going forward, developers should keep in mind health literacy when designing web portal interfaces and how test results are communicated to patients. 
Table 3 Multivariable logistic regression of patient web portal use

\begin{tabular}{|c|c|c|}
\hline Characteristic & AOR $(95 \% \mathrm{Cl})$ & $P$-value \\
\hline \multicolumn{3}{|l|}{ Demographics } \\
\hline KP (vs. UI) Site & $0.39(0.31,0.48)^{*}$ & $<0.001$ \\
\hline \multicolumn{3}{|l|}{ Age by category } \\
\hline$<65$ & $1.02(0.83,1.25)$ & 0.867 \\
\hline $65-74$ & 1 [Reference] & - \\
\hline$\geq 75$ & $0.67(0.49,0.91)^{*}$ & 0.011 \\
\hline Female (vs. male) patient & $0.80(0.62,1.05)$ & 0.105 \\
\hline Non-White (vs. White) race & $0.81(0.61,1.08)$ & 0.146 \\
\hline \multicolumn{3}{|l|}{ Educational level } \\
\hline High school graduate or less & 1 [Reference] & - \\
\hline Attended college & $1.56(1.18,2.08)^{*}$ & 0.002 \\
\hline Completed college & $1.55(1.14,2.11)^{*}$ & 0.005 \\
\hline Attended graduate school & $1.81(1.32,2.47)^{*}$ & $<0.001$ \\
\hline \multicolumn{3}{|l|}{ Patient health mindset characteristics } \\
\hline Higher health literacy & $1.5(1.09,2.07)^{*}$ & 0.014 \\
\hline \multicolumn{3}{|l|}{ Subjective numeracy } \\
\hline Higher numeracy & $1.19(0.96,1.49)$ & 0.114 \\
\hline Medium numeracy & 1 [Reference] & - \\
\hline Lower numeracy & $0.91(0.71,1.16)$ & 0.442 \\
\hline \multicolumn{3}{|l|}{ Patient Activation Score } \\
\hline Level 1 (0-42.5) & 1 [Reference] & - \\
\hline Level 2 (47.4-52.9) & $1.17(0.79,1.73)$ & 0.428 \\
\hline Level 3 (56.4-66.0) & $1.36(0.96,1.93)$ & 0.085 \\
\hline Level 4 (68.5-100) & $1.45(1.00,2.11)$ & 0.053 \\
\hline \multicolumn{3}{|l|}{ Information-seeking in health care } \\
\hline Higher level & $0.89(0.72,1.11)$ & 0.316 \\
\hline Medium level & 1 [Reference] & - \\
\hline Lower level & $0.79(0.63,1)$ & 0.054 \\
\hline \multicolumn{3}{|c|}{ Behavioral involvement in health care } \\
\hline Higher level & $1.23(0.98,1.53)$ & 0.074 \\
\hline Medium level & 1 [Reference] & - \\
\hline Lower level & $0.88(0.70,1.11)$ & 0.275 \\
\hline \multicolumn{3}{|l|}{ Health characteristics } \\
\hline \multicolumn{3}{|l|}{ History of Osteoporosis } \\
\hline Normal & 1 [Reference] & - \\
\hline Osteopenia & $1.45(1.16,1.81)^{*}$ & 0.001 \\
\hline Osteoporosis & $1.12(0.87,1.44)$ & 0.388 \\
\hline \multicolumn{3}{|l|}{ Self-rated Health } \\
\hline Poor & $0.72(0.39,1.31)$ & 0.278 \\
\hline Fair & $0.94(0.68,1.29)$ & 0.703 \\
\hline Good & 1 [Reference] & - \\
\hline Very good & $0.94(0.76,1.18)$ & 0.607 \\
\hline Excellent & $1.08(0.80,1.45)$ & 0.633 \\
\hline
\end{tabular}

Table 3 Multivariable logistic regression of patient web portal use (Continued)

\begin{tabular}{lll}
\hline Characteristic & AOR $(95 \% \mathrm{Cl})$ & $P$-value \\
\hline Number of Chronic Conditions & & \\
None & 1 [Reference] & - \\
$1-2$ & $0.92(0.76,1.12)$ & 0.426 \\
$>2$ & $0.70(0.45,1.08)$ & 0.108 \\
Provider characteristics & & \\
Intervention patient & $0.84(0.70,1.00)$ & 0.054 \\
Female Provider & $0.92(0.72,1.17)$ & 0.486 \\
Same Gender of Patient and Provider & $1.41(1.10,1.80)^{*}$ & 0.006 \\
\hline
\end{tabular}

*indicates significance of $p=0.05$ or less

Finally, there are factors that limit the generalizability of our findings. First, we included 50-64 year olds even though DXA is generally performed on older adults. In a sensitivity analysis restricted to patients $>65$ years old (data not shown), however, the associations reported here were comparable although statistical significance may not have been achieved given the reduced statistical power. Second, we relied on patient-reports of how they learned about their DXA results. Due to IRB constraints and time limitations of the two sites' information technology staff, we were unable to confirm the validity of those patient reports. Third, those who received the intervention DXA result letter may not have accessed their information via a portal because they already received their results. However, we waited four weeks after the participant had their DXA before mailing the result letter to see if they would to allow time for them to attempt to get their results in another manner. Lastly, because we could not determine the number of participants who had activated web portal accounts, the rate of those who did access their DXA results (13.9\%) may have been higher if we looked at only those with activated accounts. However, those who did not have an activated account are still considered non-users of web portals and as such did not view their DXA results on the web portal.

Patient web portals offer an efficient and quick vehicle through which clinicians can communicate with patients, and for patients to be more actively involved in their health care. Determining which patients use web portals to view their DXA results is an important step in encouraging web portal use for health communication. We found that older patients and those with less education or lower health literacy or lower levels of patient activation were less likely to use the web portal. Future research should examine the barriers and facilitators for patients accessing web portals and determine their preferences for receiving their DXA results. 


\section{Abbreviations}

Cl: Confidence Interval; CMS: Centers for Medicare and Medicaid Services; DXA: Dual-energy X-ray absorptiometry; EHR: Electronic Health Records; HIT: Health Information Technology; KPGA: Kaiser Permanente of Georgia; PAADRN: Patient Activation After DXA Result Notification; PAM: Patient Activation Measure; RA: Research Assistant; UAB: University of Alabama at Birmingham; UI: University of lowa; US: United States

\section{Acknowledgements}

We thank Sylvie Hall, BA (UI), Rebecca Burmeister, MPH (UI), Mollie Giller, MPH (UI), April Miller RT (UI), CBDT, Amna Rizvi-Toner, BA, BS (UI), Brandon Van Cleave (UI), Kara Wessels, BA (UI), Roslin Nelson (KPGA), Kathy Pines (KPGA), Aimee Khamar (KPGA), and all of the staff at the lowa Social Science Research Center for recruiting and interviewing all study participants. All except Ms. Miller were compensated from grant funds for their time. We also thank Sylvie Hall, BA (MPH), Mollie Giller, MPH (UI), Marina Reynolds, BSN (UI) for coordinating and facilitating recruitment of study participants. We also thank Xin Lu, MS, and Thuy Nguyen, MS (UI) for managing trial data. Finally, we thank the 7,749 patients who participated in PAADRN.

\section{Authors' contributions}

SE served as the project coordinator of the PAADRN study, participated in the design and coordination of the study, oversaw data collection and management, and drafted the manuscript. YL was the lead data analyst and helped to draft the manuscript. BR was the study coordinator at KPGA and assisted in the drafting of the manuscript. PC was the co-principal investigator, conceived of the study, led in its design, and helped to draft the manuscript. DR was a site principal investigator, participated in its design and coordination and helped to draft the manuscript. NC was an investigator, participated in its design and coordination and helped to draft the manuscript. KS was a site principal investigator, participated in its design and coordination and helped to draft the manuscript. FW was a co-principal investigator, led in its design and coordination and drafted the manuscript. All authors read and approved the final manuscript.

\section{Funding}

This work was supported by R01 AG033035 (Cram/Wolinsky) from the NIA at $\mathrm{NIH}$. The funding body did not have a role in the design of the study and collection, analysis, or interpretation of data and in writing the manuscript. Dr. Cram is supported by a K24 AR062133 award from NIAMS at the NIH. Dr. Edmonds is also supported through the lowa City VA Health Care System. The views expressed in this article are those of the authors and do not necessarily reflect the position or policy of the Department of Veterans Affairs or the United States government. Dr. Saag is supported by a K24 AR052361 award from the NIAMS at the NIH.

\section{Availability of data and materials}

The datasets generated and/or analysed during the current study are not publicly available as we are awaiting publication of major manuscripts but are available from the corresponding author on reasonable request. Data will be made publicly available in 2018. Please email the corresponding author for information regarding the data repository at stephanie-edmonds@uiowa.edu.

\section{Ethics approval and consent to participate}

This study was approved by the ethics committee at all three sites by the University of lowa Human Subjects Office and the Kaiser Permanente Institutional Review Board. Written consent was obtained from participants from the University of lowa and verbal consent was obtained from Kaiser Permanente participants based on policies of each institution's human subjects' office.

\section{Consent for publication}

Not applicable.

\section{Competing interests}

The authors declare that they have no competing interests with publication of this manuscript. Dr. Wright has received unrestricted grant support from Amgen for work unrelated to this project. Dr. Saag has received grants from Amgen, Eli Lilly, and Merck and has served as a paid consultant to Amgen, Eli Lilly, and Merck unrelated to this project.

\section{Author details}

'Carver College of Medicine, Department of Internal Medicine, University of lowa, 200 Newton Rd. 5231 WL, lowa City, IA 52242, USA. ${ }^{2}$ CADRE, lowa City VA Health System, lowa City, IA, USA. ${ }^{3}$ College of Public Health, Department of Biostatistics, University of lowa, lowa City, IA, USA. ${ }^{4}$ Kaiser Permanente, Center for Clinical and Outcomes Research, Atlanta, GA, USA. ${ }^{5}$ College of Medicine, Department of Internal Medicine, University of Toronto, Toronto, Canada. ${ }^{6}$ University of Toronto Health Network, Toronto, Canada.

${ }^{7}$ Mid-Atlantic Permanente Research Institute, Rockville, MD, USA. ${ }^{8}$ School of Public Health, Department of Epidemiology, University of Alabama at Birmingham, Birmingham, AL, UK. ${ }^{9}$ School of Medicine, Department of Rheumatology, University of Alabama at Birmingham, Birmingham, AL, UK. ${ }^{10}$ College of Public Health, Department of Health Management and Policy, University of lowa, lowa City, IA, USA. ${ }^{11}$ College of Nursing, University of lowa, lowa City, IA, USA.

Received: 26 October 2017 Accepted: 27 August 2019

Published online: 18 September 2019

\section{References}

1. Centers for Medicare \& Medicaid Services. Electronic Health Records (EGR) Incentive Programs Baltimore, MD2016 [updated 1/26/2016. Available from: https://www.cms.gov/Regulations-and-Guidance/Legislation/ EHRIncentivePrograms/index.html?redirect=/ehrincentiveprograms.

2. Gale BD, Bissett-Siegel DP, Davidson SJ, Juran DC. Failure to notify reportable test results: significance in medical malpractice. J Am Coll Radiol. 2011;8(11):776-9.

3. The Joint Commision. 2016 Hospital National Patient Safety Goals 2016 [Available from: http://www.jointcommission.org/assets/1/6/2016_NPSG_ HAP_ER.pdf.

4. Antinori N, Wade-Vuturo AE, Mayberry LS, Osborn CY. Secure messaging and diabetes management: experiences and perspectives of patient portal users. J Med Internet Res. 2013;20(3):519-25.

5. Harris LT, Haneuse SJ, Martin DP, Ralston JD. Diabetes quality of care and outpatient utilization associated with electronic patient-provider messaging: a cross-sectional analysis. Diabetes Care. 2009;32(7):1182-7.

6. Haun JN, Patel NR. Large-Scale Survey Findings Inform Patients' Experiences in Using Secure Messaging to Engage in Patient-Provider Communication and Self-Care Management: A Quantitative Assessment. J Med Internet Res. 2015;17(12):e282.

7. Ralston JD, Hirsch IB, Hoath J, Mullen M, Cheadle A, Goldberg HI. Webbased collaborative care for type 2 diabetes: a pilot randomized trial. Diabetes Care. 2009;32(2):234-9.

8. Zhou YY, Kanter MH, Wang JJ, Garrido T. Improved quality at Kaiser Permanente through e-mail between physicians and patients. Health affairs (Project Hope). 2010;29(7):1370-5.

9. Goldzweig CL, Towfigh AA, Paige NM, Orshansky G, Haggstrom DA, Beroes $J M$, et al. VA evidence-based synthesis program reports. Systematic review: secure messaging between providers and patients, and Patients' access to their own medical record: evidence on health outcomes, satisfaction, efficiency and attitudes. In: Washington (DC): Department of Veterans Affairs (US); 2012.

10. Otte-Trojel T, de Bont A, Rundall TG, van de Klundert J. How outcomes are achieved through patient portals: a realist review. J Am Med Inform Assoc. 2014;21(4):751-7.

11. Cram P, Rosenthal GE, Ohsfeldt R, Wallace RB, Schlechte J, Schiff GD. Failure to recognize and act on abnormal test results: the case of screening bone densitometry. Jt Comm J Qual Patient Saf. 2005;31 (2):90-7.

12. Otte-Trojel T, de Bont A, Rundall TG, van de Klundert J. What do we know about developing patient portals? A systematic literature review. J Am Med Inform Assoc. 2015;23(e1):e162-8.

13. Edmonds SW, Wolinsky FD, Christensen AJ, Lu X, Jones MP, Roblin DW, et al. The PAADRN study: a design for a randomized controlled practical clinical trial to improve bone health. Contemp Clin Trials. 2013;34(1):90-100.

14. Fagerlin A, Zikmund-Fisher BJ, Ubel PA, Jankovic A, Derry HA, Smith DM. Measuring numeracy without a math test: development of the subjective numeracy scale. Med Decis Making. 2007;27(5):672-80.

15. Krantz DS, Baum A, Wideman M. Assessment of preferences for selftreatment and information in health care. J Pers Soc Psychol. 1980;39(5):977-90 
16. Hibbard JH, Mahoney ER, Stockard J, Tusler M. Development and testing of a short form of the patient activation measure. Health Serv Res. 2005;40(6 Pt 1):1918-30.

17. Cadarette SM, Gignac MA, Beaton DE, Jaglal SB, Hawker GA. Psychometric properties of the "osteoporosis and you" questionnaire: osteoporosis knowledge deficits among older community-dwelling women. Osteoporos Int. 2007;18(7):981-9.

18. Brenneman SK, Blau EM, Chen Y, Abbott TA. Validation of a patient questionnaire, 'Osteoporosis and You', designed to assess osteoporosisrelated attitudes, knowledge and behavior. J Bone Miner Res. 2002;17(Suppl 1):S466.

19. Kim KK, Horan ML, Gendler P, Patel MK. Development and evaluation of the osteoporosis health belief scale. Res Nurs Health. 1991;14(2):155-63.

20. Horan ML, Kim KK, Gendler P, Froman RD, Patel MD. Development and evaluation of the osteoporosis self-efficacy scale. Res Nurs Health. 1998;21(5):395-403.

21. Cram P, Wolinsky FD, Lou Y, Edmonds SW, Hall SF, Roblin DW, et al. Patientactivation and guideline-concordant pharmacological treatment after bone density testing: the PAADRN randomized controlled trial. Osteoporos Int. 2016;in Press.

22. Ancker JS, Barron Y, Rockoff ML, Hauser D, Pichardo M, Szerencsy A, et al. Use of an electronic patient portal among disadvantaged populations. J Gen Intern Med. 2011;26(10):1117-23.

23. Carrell D, Ralston JD. Variation in adoption rates of a patient web portal with a shared medical record by age, gender, and morbidity level. AMIA Annu Symp Proc. 2006:871

24. Krist AH, Woolf SH, Bello GA, Sabo RT, Longo DR, Kashiri P, et al. Engaging primary care patients to use a patient-centered personal health record. Ann Fam Med. 2014;12(5):418-26.

25. Ralston JD, Hereford J, Carrell D. Use and satisfaction of a patient web portal with a shared medical record between patients and providers. AMIA Annu Symp Proc. 2006:1070.

26. Roblin DW, Houston TK 2nd, Allison JJ, Joski PJ, Becker ER. Disparities in use of a personal health record in a managed care organization. J Am Med Inform Assoc. 2009;16(5):683-9.

27. Roelofsen Y, Hendriks SH, Sieverink F, Landman GW, Groenier KH, Bilo HJ, et al. Differences between patients with type 2 diabetes mellitus interested and uninterested in the use of a patient platform (e-VitaDM-2/ZODIAC-41) J Diabetes Sci Technol. 2014:8(2):230-7.

28. Ronda MC, Dijkhorst-Oei LT, Gorter KJ, Beulens JW, Rutten GE. Differences between diabetes patients who are interested or not in the use of a patient web portal. Diabetes Technol Ther. 2013;15(7):556-63.

29. Shultz SK, Wu R, Matelski JJ, Lu X, Cram P. Patient preferences for test result notification. J Gen Intern Med. 2015:30(11):1651-6.

30. Agarwal R, Anderson C, Zarate J, Ward C. If we offer it, will they accept? Factors affecting patient use intentions of personal health records and secure messaging. J Med Internet Res. 2013;15(2):e43.

31. Longo DR. Understanding health information, communication, and information seeking of patients and consumers: a comprehensive and integrated model. Health Expect. 2005;8(3):189-94.

32. Wen KY, Kreps G, Zhu F, Miller S. Consumers' perceptions about and use of the internet for personal health records and health information exchange: analysis of the 2007 health information National Trends Survey. J Med Internet Res. 2010;12(4):e73.

\section{Publisher's Note}

Springer Nature remains neutral with regard to jurisdictional claims in published maps and institutional affiliations.

Ready to submit your research? Choose BMC and benefit from:

- fast, convenient online submission

- thorough peer review by experienced researchers in your field

- rapid publication on acceptance

- support for research data, including large and complex data types

- gold Open Access which fosters wider collaboration and increased citations

- maximum visibility for your research: over $100 \mathrm{M}$ website views per year

At BMC, research is always in progress.

Learn more biomedcentral.com/submissions 